\title{
In Vitro Chemosensitivity Test for Gastric Cancer Specimens Predicts Effectiveness of Oxaliplatin and 5-Fluorouracil
}

\author{
YOSHIKAZU KANAZAWA, TAKESHI YAMADA, ITSUO FUJITA, DAISUKE KAKINUMA, \\ KUNIHIKO MATSUNO, HIROKI ARAI, TOMOHIRO SHIMODA, KAZUHIDE KO, SHUNJI KATO, \\ TAKESHI MATSUTANI, NOBUTOSHI HAGIWARA, TSUTOMU NOMURA and EIJI UCHIDA
}

Department of Gastrointestinal and Hepato-Billiary-Pancreatic Surgery, Nippon Medical School, Tokyo, Japan

\begin{abstract}
Aim: Cisplatin plus 5-fluorouracil (5-FU) or $S-1$ is a standard therapy for gastric cancer $(G C)$. However, cisplatin is emetic and potentially nephrotoxic. Oxaliplatin may be less toxic, but few basic data are available for this setting. Here, we evaluated oxaliplatin for GC, by testing surgical specimens. Materials and Methods: We evaluated effects of oxaliplatin and 5-FU, alone and in combination, on surgical specimens from 11 patients with GC, using collagen gel droplet embedded culture drug tests. Results: Oxaliplatin was less efficacious than 5-FU, and its synergistic effect was less in tumors highly sensitive to 5-FU than in those with low sensitivity. Tumor differentiation and drug sensitivity were not correlated. Conclusion: Although oxaliplatin monotherapy had little effect on $G C$, we saw a limited synergistic effect of oxaliplatin with 5-FU in 5-FUsensitive patients. Collagen gel droplet embedded culture drug tests may predict this synergistic effect, and help select candidates for this or other regimens.
\end{abstract}

S-1 is an important therapeutic agent for advanced gastric cancer (GC) (1), and as adjuvant chemotherapy for GC postoperatively $(2,3)$. However, some patients need more powerful antitumor effects. The SPIRITS trial in 2008 showed that adding cisplatin to S-1 led to longer overall (OS) and progression-free (PFS) survival than S-1 alone (2); this combination, or cisplatin with fluorouracil $(5-\mathrm{FU})$ is now a standard first-line chemotherapy regimen (4-6). However, platinum-containing agents have a strong emetic effect, and

Correspondence to: Yoshikazu Kanazawa, Department of Gastrointestinal and Hepatobiliary-Pancreatic Surgery, Nippon Medical School, 1-1-5 Sendagi, Bunkyo-ku, Tokyo 113-8603, Japan. Tel: +81 338222131, Fax: +81 356850989, e-mail: kanazawa-y@nms.ac.jp

Key Words: Gastric cancer, CD-DST, synergetic effect, oxaliplatin, 5 -FU. can cause sensory neuropathy and nephrotoxicity, which complicate their use in outpatients. Oxaliplatin, like cisplatin, is a platinum-containing agent, but has no nephrotoxic effects. With fluorouracil and leucovorin or S-1, oxaliplatin is reportedly an effective agent against colorectal cancer (79), and advanced GC. In fact, a regimen of S-1 plus oxaliplatin has been shown not to be inferior to that of S-1 plus cisplatin with regard to PFS $(10,11)$.

$\mathrm{S}-1$ is the most effective agent in GC treatment and is an important key drug (1). Thus in selection of drug to be added in combination with $\mathrm{S}-1$, it is necessary to be careful. Although the enhancing effect of platinum-containing agents on 5-FU and S-1 has been shown through clinical trials, adverse effects from these combinations often curtail their use $(5,12,13)$.

Although one in vitro study used five established cell lines to evaluate the effect of oxaliplatin alone on GC (14), we know of no studies that evaluated the synergistic effect of 5-FU and oxaliplatin using clinical samples. Therefore, in this study, we evaluated the additional efficacy of oxaliplatin when added to 5-FU, using an in vitro chemosensitivity test on resected GC specimens.

\section{Materials and Methods}

Patients. We used specimens resected from 11 patients who underwent surgery for advanced GC, eight males and three females (age range: 49-88 years), from July 2014 to March 2016. Their clinicopathological characteristics are summarized in Table I.

Clinical samples. Among the 11 specimens there were five differentiated adenocarcinomas and six undifferentiated adenocarcinomas. We obtained the primary tumor cells by stripping them off the surfaces of cancerous specimens at the end of surgery as empirical data suggest that the largest number of cancer cells can be collected from tissue surfaces (15). All samples were irrigated 10 times, with $40 \mathrm{ml}$ of saline each time, without antibiotics. After irrigation, the samples were stored in Eagle's minimal essential medium (Gibco ${ }^{\circledR}$, Thermo-Fisher Scientific, Waltham, MA, USA) at $4^{\circ} \mathrm{C}$ until chemosensitivity testing. 
Table I. Patient and tumor characteristics.

\begin{tabular}{|c|c|c|c|c|c|c|c|c|c|}
\hline Patient & Age, years & Gender & Histological type & $\mathrm{T}$ & $\mathrm{N}$ & $\mathrm{H}$ & $\mathrm{P}$ & M & Stage \\
\hline 1 & 85 & $\mathrm{~F}$ & Differentiated & $4 a$ & 0 & 0 & 0 & 0 & IIB \\
\hline 2 & 69 & M & Undifferentiated & $4 a$ & 1 & 0 & 1 & 0 & IV \\
\hline 3 & 60 & $\mathrm{~F}$ & Undifferentiated & $4 a$ & 2 & 0 & 1 & 0 & IV \\
\hline 4 & 49 & M & Differentiated & $4 b$ & 2 & 1 & 0 & 0 & IIIC \\
\hline 5 & 51 & M & Undifferentiated & $4 \mathrm{a}$ & 0 & 0 & 0 & 0 & IIB \\
\hline 6 & 68 & $\mathrm{~F}$ & Differentiated & 3 & 2 & 0 & 0 & 0 & IIIA \\
\hline 7 & 77 & M & Undifferentiated & $4 a$ & 2 & 1 & 0 & 1 & IV \\
\hline 8 & 82 & M & Differentiated & $4 \mathrm{~b}$ & 2 & 0 & 1 & 0 & IV \\
\hline 9 & 88 & M & Undifferentiated & 3 & 2 & 0 & 0 & 0 & IIIA \\
\hline 10 & 74 & M & Differentiated & 3 & 0 & 0 & 0 & 0 & IIA \\
\hline 11 & 74 & M & Undifferentiated & $4 \mathrm{a}$ & 3 & 0 & 0 & 1 & IV \\
\hline
\end{tabular}

F: Female; M: male; T: depth of tumor invasion; 3: tumor invades the subserosa (SS); 4a: tumor invasion is contiguous to the serosa or penetrates the serosa (SE); 4b: tumor invades adjacent structures (SI); N: lymph node metastasis; 0: no regional lymph node metastasis; 1: metastasis in 1-2 regional lymph nodes; 2: Metastasis in 3-6 regional lymph nodes; 3: metastasis in 7 or more regional lymph nodes; H: hepatic metastasis; 0: no hepatic metastasis, 1: hepatic metastasis; P: peritoneal metastasis; 0: no peritoneal metastasis; 1: peritoneal metastasis; M: presence or absence and sites of distant metastasis; 0: no distant metastasis; 1: distant metastasis; Japanese classification of gastric carcinoma: 3rd English edition.

In vitro chemosensitivity test. The collagen gel droplet embedded culture drug test (CD-DST) was carried out according to the method reported by Kobayashi et al. (15-17). A portion from each tumor specimen was excised and sliced into thin sections. Each sample was treated with a dispersed enzyme cocktail EZ (Primaster ${ }^{\circledR}$ Reagent; Kurabo Industries, Osaka, Japan). The obtained cell suspensions were inoculated into collagen-coated flasks (CG Flasks; Kurabo Industries) and cultured in PCM-1 pre-culture medium (Primaster ${ }^{\circledR}$ Content) containing $10 \%$ fetal bovine serum at $37^{\circ} \mathrm{C}$ in $5 \% \mathrm{CO}_{2}$ overnight. Next, the collagen gel was digested with $0.05 \%$ EZ and viable cancer cells were obtained. Type I collagen (Cellmatrix Type CD; Kurabo Industries Ltd. Osaka, Japan), 10x concentrated F-12 medium, and reconstitution buffer were mixed together in ice water at a ratio of 8:1:1 (Primaster $_{\overparen{B}}$ content). The prepared cancer cell suspensions were each added to the collagen solution at final densities of $1 \times 10^{5}$ cells $/ \mathrm{ml}$. Subsequently, the tumor cells in the collagen gel droplets were exposed to the treatment drugs at concentrations corresponding to the area under the curve (AUC) for drug concentration and time. Three drops of the collagen-cell mixture ( $30 \mu \mathrm{l} / \mathrm{droplet})$ were placed in each well of a 6-well multiple plate on ice and allowed to gel at $37^{\circ} \mathrm{C}$ in a $\mathrm{CO}_{2}$ incubator; the final concentration was approximately $3 \times 10^{3}$ cells per collagen gel droplet. Dulbecco's modified Eagle's medium and F12 medium (Gibco) containing 10\% fetal bovine serum was overlaid on each well $1 \mathrm{~h}$ later and plates were incubated in a $\mathrm{CO}_{2}$ incubator at $37^{\circ} \mathrm{C}$ overnight. The in vitro chemosensitivity of these samples to oxaliplatin, 5-FU and combination of oxaliplatin and 5-FU was analyzed by CD-DST. The oxaliplatin concentration was $0.6 \mu \mathrm{g} / \mathrm{ml}$, and that of 5-FU was $1 \mu \mathrm{g} / \mathrm{ml}$ (17). Contact time for the drugs was 24 hours. The AUC for oxaliplatin at $60-130 \mathrm{mg} / \mathrm{m}^{2}$ is $17.3 \mu \mathrm{g} \mathrm{h} / \mathrm{ml}$ (18). The AUC of our study was $14.4-17.3 \mu \mathrm{g} \mathrm{h} / \mathrm{ml}$. After removing the medium containing the anticancer drugs, each well was rinsed twice with $3 \mathrm{ml}$ Hanks' balanced salt solution, overlaid with $4 \mathrm{ml}$ PCM-2 medium (Primaster ${ }^{\circledR}$ content, serum-free medium), and incubated for another 7 days. At the end of the incubation, a neutral red solution (Wako Pure Chemical Industries, Ltd., Osaka Japan) was added to each well to a final concentration of $50 \mu \mathrm{g} / \mathrm{ml}$, and
Table II. Results of chemosensitivity tests on gastric cancer specimens. The in vitro chemosensitivity effect of 5-fluorouracil (5-FU) and oxaliplatin, alone and in combination, was expressed as the inhibitory rate $(I R)$, which was calculated using the following formula: $I R(\%)=$ $(C-T) / C \times 100$, where $T$ was total colony volume of the treated group and $C$ was that of the untreated control group. A combination/5-FU ratio $\geq 1.2$ indicates a positive synergistic effect.

\begin{tabular}{lcccc}
\hline Patient & 5-FU & Oxaliplatin & Combination & $\begin{array}{c}\text { Ratio (combination/ } \\
\text { 5-FU) }\end{array}$ \\
\hline 1 & & & & 1.11 \\
2 & 19.1 & 0 & 21.2 & 1.86 \\
3 & 17.2 & 4.5 & 32.0 & 0.81 \\
4 & 18.0 & 11.2 & 14.6 & 0.91 \\
5 & 50.4 & 17.4 & 46.0 & 1.01 \\
6 & 42.9 & 18.5 & 43.4 & 0.42 \\
7 & 9.5 & 6.2 & 4.0 & 2.97 \\
8 & 6.8 & 16.4 & 20.2 & 1.06 \\
9 & 45.2 & 25.6 & 47.9 & 1.16 \\
10 & 27.1 & 32.4 & 31.5 & 1.25 \\
11 & 40.7 & 27.8 & 50.8 & 1.30 \\
\hline
\end{tabular}

Combination: 5-FU plus oxaliplatin.

cancer cell colonies in the collagen gel droplets were stained for $2 \mathrm{~h}$. Each collagen droplet was fixed with $10 \%$ neutral buffered formalin, washed in water, air dried, and quantified using image analysis (Primage System, Solution Systems Inc., -Tokyo, Japan). This sensitivity test was conducted at LSI Medience Corporation laboratory (Tokyo, Japan).

The in vitro chemosensitivity effect was expressed as the inhibitory rate (IR), which was calculated by the following formula: IR $(\%)=(\mathrm{C}-\mathrm{T}) / \mathrm{C} \times 100$, where $\mathrm{T}$ was total colony volume of the treated group and $\mathrm{C}$ was that of the untreated control group. To quantify the effect of the combination regimen, we used the 


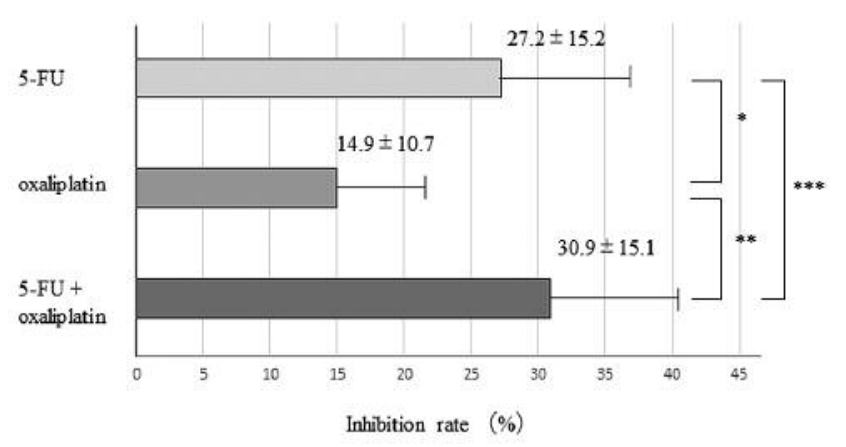

Figure 1. Inhibitory rates using 5-fluorouracil (5-FU), oxaliplatin, and 5-FU in combination with theoxaliplatin. Data are shown as the mean $\pm S D$. Significantly different at $* p=0.0095$, ** $p=0.0014$ and $* * * p=0.1035$.

following ratio: combination/5-FU. We considered a ratio $\geq 1.2$ to indicate a positive synergistic effect.

\section{Results}

Table II shows the IRs for 5-FU, oxaliplatin, and their combination for each of the 11 patient specimens. Whereas IR values varied for 5-FU, those for oxaliplatin were low overall, and significantly lower than those for 5-FU $(p=0.0095)$ and the combination regimen $(p=0.0014)$. However, the IRs for 5-FU and the combination did not significantly differ $(p=0.1035$; Figure 1$)$.

Synergistic effects were observed in four out of 11 cases (36.3\%; patients 2, 7, 10 and 11) based on IRs for 5-FU. Four patients (patiens $4,5,8$ and 10) were highly sensitive to 5 -FU (mean IR $\geq 27.2 \%$ ), among whom only patient 10 showed a synergistic effect from oxaliplatin. Among the five patients not responding to 5-FU (patients 1, 2, 3, 6, 7 and 11), we observed synergetic effects in three (patients 2,7 and 11). We did not see a significant correlation between tumor differentiation and IR using 5-FU or oxaliplatin, or their combination (Table III).

\section{Discussion}

In this study, we evaluated the synergistic effect of 5-FU and oxaliplatin using an in vitro chemosensitivity test on surgical GC specimens. We had three novel findings: (i) the synergic effect of the combination of oxaliplatin and 5-FU is limited in vitro; (ii) among patients whose $\mathrm{GC}$ is sensitive to $5-\mathrm{FU}$, additional synergistic effects from adding oxaliplatin to 5FU are unlikely; and (iii) tumor differentiation does not correlate with synergetic effects of these drugs.

The synergistic effect of oxaliplatin with 5-FU is limited in vitro, and (as described above), oxaliplatin alone had little effect on GC in this in vitro study. Only four patients showed
Table III. Inhibitory rates (\%) by histological type and treatment with 5-fluorouracil (5-FU), oxaliplatin and their combination.

\begin{tabular}{lcccc}
\hline Histological type & $\mathrm{n}$ & 5-FU & Oxaliplatin & Combination \\
\hline Differentiated & 5 & $33.0 \pm 17.7$ & $15.4 \pm 12.1$ & $34.0 \pm 20.5$ \\
Undifferentiated & 6 & $22.3 \pm 12.1$ & $14.6 \pm 10.5$ & $28.4 \pm 10.1$ \\
$p$-Value & & 0.27 & 0.91 & 0.53 \\
\hline
\end{tabular}

synergistic response to oxaliplatin combined with $5-\mathrm{FU}$ in this study. Whereas IRs varied for 5-FU among our 11 patients, the IRs for oxaliplatin were low as a whole. Thus, in GC, the antitumor effect of oxaliplatin is expected to have a synergistic effect with 5-FU.

Although the response rate of ovarian cancer to oxaliplatin has been reported to be $29 \%$ (19), the reported response of colorectal cancer was only $10 \%$ (20).

The synergistic effect between oxaliplatin and 5-FU has been observed in colon, breast and ovarian cancer in vivo and in vitro $(21,22)$, but the mechanism that underlies their interaction is unclear. Moreover, identifying patients who can benefit from this combination is difficult. Among patients with advanced colorectal cancer, PFS and OS were reportedly equivalent in the FOLOFOX7 regimen, which has a modified oxaliplatin schedule, to those in the standard FOLOFOX4 regimen. The FOLFOX 7 is a modified regimen and the administration schedule of oxaliplatin as Stop-andGo has been changed according to adverse events. Furthermore, patients with colorectal cancer who respond well to FOLFOX are also expected to respond well to 5-FU plus leucovorin without oxaliplatin (23). Therefore, although addition of oxaliplatin to a standard regimen may be beneficial, such patients should be personally assessed.

Tumor differentiation did not correlate with response to 5FU, oxaliplatin or their combination. Although Eriguchi et al. reported poorly differentiated colorectal cell lines to be the most sensitive to oxaliplatin (14), as far as we are aware, no clinical trial has shown an effect of oxaliplatin with regard to tumor differentiation (24-28).

Furthermore, which patients can benefit from the addition of oxaliplatin is not known, and no basic data have been reported to predict responses to combination therapy. Even in our study, less-differentiated adenocarcinoma did not appear to respond well to oxaliplatin. However, addition of oxaliplatin is recommended in clinical trials, so it should not be omitted from a patient's regimen without clear reasons for doing so.

CD-DST uses a three-dimensional culture embedded in type I collagen gel and, unlike other chemosensitivity tests, antitumor activity is evaluated at drug concentrations similar to those obtained in humans (15-17). We, therefore, consider 
CD-DST to be the best choice for evaluating synergistic drug effects using specimens in vitro.

This study had several limitations. Firstly, the sample size was small. However, we used genuine clinical samples as opposed to cell lines; this is a strength of this study. Secondly, because the number of clinical samples was limited, drug effects were evaluated only for certain concentrations and contact times, and other variables could not be tested. However, the AUCs in this study were set as they would be with clinical settings. Thirdly, for ethical reasons, this was inevitably an experimental study in vitro and was not reflected in patients' treatments. Nevertheless, this study potentially offers a means of assessing the appropriateness of treatment for individual patients for more personalized management of GC.

In conclusion, limited synergistic effects of oxaliplatin may be expected in 5-FU-responsive patients. Candidates for 5-FU monotherapy or 5-FU combined with oxaliplatin should be carefully selected; use of CD-DST may help predict response to such therapy.

\section{Informed Consent}

Informed consent was obtained from all individual participants included in the study.

\section{Conflicts of Interest}

The Authors declare that they have no conflict of interest.

\section{References}

1 Koizumi W, Kurihara M, Nakano S and Hasegawa K: Phase II study of S-1, a novel oral derivative of 5-fluorouracil, in advanced gastric cancer. For the S-1 Cooperative Gastric Cancer Study Group. Oncology 58: 191-197, 2000.

2 Sakuramoto S, Sasako M, Yamaguchi T, Kinoshita T, Fujii M, Nashimoto A, Furukawa H, Nakajima T, Ohashi Y, Imamura H, Higashino M, Yamamura Y, Kurita A Arai K and the ACTS-GC Group: Adjuvant chemotherapy for gastric cancer with S-1, an oral fluoropyrimidine. N Engl J Med 357: 1810-1820, 2007.

3 Sasako M, Sakuramoto S, Katai H, Kinoshita T, Furukawa H, Yamaguchi T, Nashimoto A, Fujii M, Nakajima T and Ohashi Y: Five-year outcomes of a randomized phase III trial comparing adjuvant chemotherapy with S-1 versus surgery alone in stage II or III gastric cancer. J Clin Oncol 29: 4387-4393, 2011.

4 Kang YK, Kang WK, Shin DB, Chen J, Xiong J, Wang J, Lichinitser M, Guan Z, Khasanov R, Zheng L, Philco-Salas M, Suarez T, Santamaria J, Forster G and McCloud PI: Capecitabine/cisplatin versus 5 -fluorouracil/cisplatin as first-line therapy in patients with advanced gastric cancer: a randomised phase III noninferiority trial. Ann Oncol 20: 666-673, 2009.

5 Koizumi W , Narahara H, Hara T, Takagane A, Akiya T, Takagi M, Miyashita K, Nishizaki T, Kobayashi O, Takiyama W, Toh Y, Nagaie T, Takagi S, Yamamura Y, Yanaoka K, Orita $\mathrm{H}$ and Takeuchi M: S-1 plus cisplatin versus S-1 alone for first-line treatment of advanced gastric cancer (SPIRITS trial): a phase III trial. Lancet Oncol 9: 215-221, 2008.
6 Ajani JA, Rodriguez W, Bodoky G, Moiseyenko V, Lichinitser M, Gorbunova V, Vynnychenko I, Garin A, Lang I and Falcon S: Multicenter phase III comparison of cisplatin/S-1 with cisplatin/infusional fluorouracil in advanced gastric or gastroesophageal adenocarcinoma study: The FLAGS Trial. J Clin Oncol 28: 1547-1553, 2010.

7 De Gramont A, Figer A, Seymour M, Homerin M, Hmissi A, Cassidy J, Boni C, Cortes-Funes H, Cervantes A, Freyer G, Papamichael D, Le Bail N, Louvet C, Hendler D, de Braud F, Wilson C, Morvan F and Bonetti A: Leucovorin and fluorouracil with or without oxaliplatin as first-line treatment in advanced colorectal cancer. J Clin Oncol 18: 2938-2947, 2000.

8 Colucci G, Gebbia V, Paoletti G, Giuliani F, Caruso M, Gebbia N, Cartenì G, Agostara B, Pezzella G, Manzione L, Borsellino N, Misino A, Romito S, Durini E, Cordio S, Di Seri M, Lopez M, Maiello E, Montemurro S, Cramarossa A, Lorusso V, Di Bisceglie M, Chiarenza M, Valerio MR, Guida T, Leonardi V, Pisconti S, Rosati G, Carrozza F, Nettis G, Valdesi M, Filippelli G, Fortunato S, Mancarella S, Brunetti C and the Gruppo Oncologico Dell'Italia Meridionale: Phase III randomized trial of FOLFIRI versus FOLFOX4 in the treatment of advanced colorectal cancer: a multicenter study of the Gruppo Oncologico Dell'Italia Meridionale. J Clin Oncol 23: 4866-4875, 2005.

9 Zang DY, Lee BH, Park HC, Song HH, Kim HJ, Jung JY, Kim JH, Kim HY, Kwon JH, Hwang SW, Park SR, Park CH, Kim KO, Kim MJ and Jang KM: Phase II study with oxaliplatin and S-1 for patients with metastatic colorectal cancer. Ann Oncol 20: 892-896, 2009.

10 Hamada C, Yamada Y, Azuma M, Nishikawa K, Gotoh M, Bando H, Sugimoto N, Nishina T, Amagai K, Chin K, Niwa Y, Tsuji A, Imamura H, Tsuda M, Yasui H, Fujii H, Yamaguchi K, Yasui H, Hironaka S, Shimada K, Miwa H and Hyodo I: Metaanalysis supporting noninferiority of oxaliplatin plus S-1 to cisplatin plus S-1 in first-line treatment of advanced gastric cancer (G-SOX study): indirect comparison with S-1 alone. Int J Clin Oncol 21: 668-675, 2016.

11 Hironaka S, Sugimoto N, Yamaguchi K, Moriwaki T, Komatsu Y, Nishina T, Tsuji A, Nakajima TE, Gotoh M, Machida N, Bando H, Esaki T, Emi Y, Sekikawa T, Matsumoto S, Takeuchi M, Boku N, Baba H and Hyodo I: S-1 plus leucovorin versus S1 plus leucovorin and oxaliplatin versus S-1 plus cisplatin in patients with advanced gastric cancer: a randomised, multicentre, open-label, phase 2 trial. Lancet Oncol 17: 99-108, 2016.

12 Kris MG, Hesketh PJ, Somerfield MR, Feyer P, Clark-Snow R, Koeller JM, Morrow GR, Chinnery LW, Chesney MJ, Gralla RJ and Grunberg SM: American Society of Clinical Oncology guideline for antiemetics in oncology: update 2006. J Clin Oncol 24: 2932-2947, 2006.

13 Arany I and Safirstein RL: Cisplatin nephrotoxicity. Semin Nephrol 23: 460-464, 2003.

14 Eriguchi M, Nonaka Y, Yanagie H, Yoshizaki I, Takeda Y and Sekiguchi M: A molecular biological study of anti-tumor mechanisms of an anti-cancer agent oxaliplatin against established human gastric cancer cell lines. Biomed Pharmacother 57: 412$415,2003$.

15 Kobayashi H, Tanisaka K, Doi O, Kodama K, Higashiyama M, Nakagawa H, Miyake M, Taki T, Hara S, Yasutomi M, Hanatani Y, Kotake K and Kubota T: An in vitro chemosensitivity test for solid human tumors using collagen gel droplet embedded cultures. Int J Oncol 11: 449-455, 1997. 
16 Kobayashi H: Development of a new in vitro chemosensitivity test using collagen gel droplet embedded culture and image analysis for clinical usefulness. Recent Results Cancer Res 161: 48-61, 2003

17 Kobayashi H, Higashiyama M, Minamigawa K, Tanisaka K, Takano T, Yokouchi H, Kodama K and Hata T: Examination of in vitro chemosensitivity test using collagen gel droplet culture method with colorimetric endpoint quantification. Jpn J Cancer Res 92: 203-210, 2001.

18 Nikanjam M, Stewart CF, Takimoto CH, Synold TW, Beaty O, Fouladi $\mathrm{M}$ and Capparelli EV: Population pharmacokinetic analysis of oxaliplatin in adults and children identifies important covariates for dosing. Cancer Chemother Pharmacol 75: 495503, 2015.

19 Chollet P, Bensmaine MA, Brienza S, Deloche C, Cure H, Caillet $\mathrm{H}$ and Cvitkovic E: Single agent activity of oxaliplatin in heavily pretreated advanced epithelial ovarian cancer. Ann Oncol 7: 1065-1070, 1996.

20 Machover D, Diaz-Rubio E, de Gramont A, Schilf A, Gastiaburu JJ, Brienza S, Itzhaki M, Metzger G, N'Daw D, Vignoud J, Abad A, Francois E, Gamelin E, Marty M, Sastre J, Seitz JF and Ychou M: Two consecutive phase II studies of oxaliplatin (LOHP) for treatment of patients with advanced colorectal carcinoma who were resistant to previous treatment with fluoropyrimidines. Ann Oncol 7: 95-98, 1996.

21 Raymond E, Buquet-Fagot C, Djelloul S, Mester J, Cvitkovic E, Allain P, Louvet $\mathrm{C}$ and Gespach C: Antitumor activity of oxaliplatin in combination with 5-fluorouracil and the thymidylate synthase inhibitor AG337 in human colon, breast and ovarian cancers. Anticancer Drugs 8: 876-885, 1997.

22 Raymond E, Chaney SG, Taamma A and Cvitkovic E. Oxaliplatin: a review of preclinical and clinical studies. Ann Oncol 9: 1053-1071, 1998.

23 Tournigand C, Cervantes A, Figer A, Lledo G, Flesch M, Buyse M, Mineur L, Carola E, Etienne PL, Rivera F, Chirivella I, PerezStaub N, Louvet C, André T, Tabah-Fisch I and de Gramont A: OPTIMOX1: a randomized study of FOLFOX4 or FOLFOX7 with oxaliplatin in a stop-and-go fashion in advanced colorectal cancer - a GERCOR study. J Clin Oncol 24: 394-400, 2006.
24 Al-Batran SE, Atmaca A, Hegewisch-Becker S, Jaeger D, Hahnfeld S, Rummel MJ, Seipelt G, Rost A, Orth J, Knuth A and Jaeger E: Phase II trial of biweekly infusional fluorouracil, folinic acid, and oxaliplatin in patients with advanced gastric cancer. J Clin Oncol 22: 658-663, 2004.

25 Al-Batran SE, Hartmann JT, Probst S, Schmalenberg H, Hollerbach S, Hofheinz R, Rethwisch V, Seipelt G, Homann N, Wilhelm G, Schuch G, Stoehlmacher J, Derigs HG, HegewischBecker S, Grossmann J, Pauligk C, Atmaca A, Bokemeyer C, Knuth A, Jäger $\mathrm{E}$ and Arbeitsgemeinschaft Internistische Onkologie: Phase III Trial in metastatic gastroesophageal adenocarcinoma with fluorouracil, leucovorin plus either oxaliplatin or cisplatin: a study of the Arbeitsgemeinschaft Internistische Onkologie. J Clin Oncol 26: 1435-1442, 2008.

26 Chao Y, Yeh KH, Chang CJ, Chen LT, Chao TY, Wu MF, Chang CS, Chang JY, Chung CY, Kao WY, Hsieh RK and Cheng AL: Phase II study of weekly oxaliplatin and 24-h infusion of highdose 5-fluorouracil and folinic acid in the treatment of advanced gastric cancer. Br J Cancer 91: 453-458, 2004.

27 Park YH, Park YH1, Kim BS, Ryoo BY and Yang SH: A phase II study of capecitabine plus 3-weekly oxaliplatin as first-line therapy for patients with advanced gastric cancer. Br J Cancer 94: 959-963, 2006.

28 Lordick F, Lorenzen S, Stollfuss J, Vehling-Kaiser U, Kullmann F, Hentrich M, Zumschlinge R, Dietzfelbinger H, Thoedtmann J, Hennig M, Seroneit T, Bredenkamp R, Duyster J and Peschel C: Phase II study of weekly oxaliplatin plus infusional fluorouracil and folinic acid (FUFOX regimen) as first-line treatment in metastatic gastric cancer. Br J Cancer 93: 190-194, 2005.
Received June 9, 2017

Revised July 10, 2017

Accepted July 12, 2017 